\title{
Neuere Entwicklungen im türkischen Straf- und Strafprozessrecht im Lichte der EMRK
}

\begin{abstract}
Summary
In the past decade, Turkey has undergone a transformation which not only brought an enormous economic upswing to the country, but also made fundamental reforms to the legal system possible. At the beginning, the EU rapprochement may have represented an important driving force in this process, but in the meantime, the will to reform has been uncoupled from this vague EU perspective and displays its own momentum. However, those who apply these laws in the practice are still far from implementing the impressive legislation. Recently, the Turkish parliament once again passed two important legislative packages dealing largely with criminal law which are of particular import for the free organization of the legal system and which explicitly refer to the guidelines of the ECHR and ECtHR jurisprudence. In the following article, these recent developments will be sketched out and weak points in the practical application will be described where they are present.
\end{abstract}

\section{Résumé}

Au cours de la dernière décennie, la Turquie a connu une profonde transformation qui a non seulement généré un extraordinaire essor économique dans le pays, mais aussi permis d'introduire des réformes fondamentales dans son système juridique. Dans un premier temps, le rapprochement avec l'Union européenne a sans doute été un moteur important dans ce processus; entre-temps toutefois, la volonté de réforme s'est dissociée de cette vague perspective européenne et a révélé une dynamique propre. Néanmoins, en pratique, les progrès considérables réalisés sont encore loin d'être mis en œuvre dans la législation. Récemment, le parlement turc a à nouveau adopté deux importants paquets législatifs, ayant essentiellement trait au droit pénal, qui revêtent une importance considérable s'agissant de l'organisation libérale du système juridique turc et qui renvoient expressément aux dispositions de la CEDH et à la jurisprudence de la CtEDH. $L$ 'article suivant sera consacré à ces récents développements ainsi qu'aux faiblesses de la mise en œuvre pratique aux points concernés.

Die rasante Entwicklung der Türkei im letzten Jahrzehnt hat das Land nicht nur zu einem begehrten Wirtschaftsstandort gemacht, sondern auch tiefgreifende Änderungen im Rechtssystem herbeigeführt, die die Grundlage einer „stillen Revolution“ gebildet haben. Insbesondere im Strafrecht waren im Zuge der EU-Annäherung umwälzende Änderungen zu verzeichnen: Ein vollständig neu aufgestelltes Strafgesetzbuch, eine ebenso grunderneuerte Strafprozessordnung sowie eine an Menschenrechtsstandards orientierte Strafvollzugsordnung bescherten der Türkei eine normative Basis für ein Strafrechts- 
system, das den Anschluss an die modernen Strömungen in der westlichen Welt ermöglicht. ${ }^{1}$ Allerdings zeigte sich bald, dass dieser Paradigmenwechsel ohne eine entsprechende Umsetzung in der Praxis nicht viel wert ist. Ebenso war klar, dass die Konstituierung einer freiheitlichen Strafrechtsordnung ein evolutionärer Prozess ist, der eine Daueraufgabe darstellt. In diesem Sinne befindet sich die Türkei weiterhin in einem stetigen Wandel in Gesetzgebung und Praxis, der durch aktuelle Entwicklungen in der Politik (Stichworte: Lösung der Kurdenproblematik, neue Verfassung, Modernisierung des Justizapparats) einerseits befeuert wird, andererseits aber auch ins Stocken geraten kann („Gezi-Park“). In jüngerer Zeit hat das türkische Parlament zwei weitere wichtige „Justizpakete“ (Yarg1 Paketi) verabschiedet, die in dieser Hinsicht bedeutende Elemente enthalten und sich dabei gezielt auf die Vorgaben der EMRK beziehen. Ziel dieses Beitrags ist es, anhand einer Auswahl aus diesen Neuerungen einen Überblick über den aktuellen Reformstand in der Türkei zu geben.

\section{Materielles Strafrecht}

Die Bedeutung der EMRK ${ }^{2}$ für die jüngsten Reformbestrebungen geht bereits aus dem Titel des Vierten Justizpakets ${ }^{3}$ hervor: „Gesetz über die Änderung einiger Gesetze im Zusammenhang mit Menschenrechten und der Meinungsfreiheit." Gelegentlich nutzt der türkische Gesetzgeber die Überschriften seiner legislativen Akte für eine Manifestation einer Grundhaltung; in diesem Falle hat er ersichtlich den sog. „Lösungsprozess“ (Çözüm Süreci) im Blick, der im Spätherbst 2012 mit dem Ziel angestoßen wurde, die jahrzehntelange Kurdenproblematik auf friedlichem Wege zu lösen. Mit dieser neuen Ära, deren Erfolg bisweilen am seidenen Faden zu hängen scheint, verbindet sich eine stärkere Betonung der Gemeinsamkeiten der Ethnien in der Türkei bei Aufgabe des stringenten türkischen Nationalismus und die Abkehr vom Feindbild „Kurdisches Volk“ bzw. die Differenzierung zwischen der Terrororganisation PKK und den legitimen Interessen des kurdischen Bevölkerungsanteils.

In der Frage der Meinungsfreiheit war bereits bedeutsam, dass die vom EGMR mit Verweis auf Art. 10 Abs. 1 EMRK vielfach beanstandete ${ }^{4}$ Bestimmung des Art. 8 des

1 Siehe dazu Roxin/Isfen GA 2005, 228 ff., ferner Isfen Das Schuldprinzip im Strafrecht unter besonderer Berücksichtigung des türkischen Rechts 2008, S. 12 ff.; Tellenbach KAS/Auslandsinformationen $4 / 05$, S. $78 \mathrm{ff}$.

2 Eine grundlegende Auseinandersetzung mit der „Menschenrechtsproblematik der Türkei im Lichte der Europäischen Menschenrechtskonvention“ liefern Tezcan/Erdem/Sancaktar Avrupa İnsan Hakları Sözleşmesi Işı̆ğında Türkiye'nin İnsan Hakları Sorunu, 2. Aufl. 2004, passim.

3 Gesetz mit der Nr. 6459 vom 11.4.2013, veröffentlicht in Resmî Gazete vom 30.4.2013, Nr. 28633.

4 Zum Beispiel Okçuoğlu vs. Türkei, Urteil vom 9.7.1999, Individualbeschwerde-Nr. 24246/94; Erdoğdu und Ince vs. Türkei, Urteil vom 9.7.1999, Individualbeschwerde-Nr. 25067/94 und 25068/94; Gerger vs. Türkei, Urteil vom 9.7.1999, Individualbeschwerde-Nr. 24919/94; Şener vs. Türkei Urteil vom 11.9.1999, Individualbeschwerde-Nr. 26680/95. Weitere Beispiele aus der EGMR-Rechtsprechung, die die Meinungsfreiheit in der Türkei betreffen, finden sich bei Eryılmaz Polis Bilimleri Dergisi, 7-8/2000, S. 305 ff.; siehe ferner ders. Ankara Barosu Dergisi 1/2001, S. 249 ff. 
Terrorbekämpfungsgesetzes ${ }^{5}$ (TBG) im Zuge der EU-Annäherung restlos abgeschafft wurde. ${ }^{6}$ Diese Vorschrift - auch als „Intellektuellendelikt“" ${ }^{67}$ bekannt - untersagte in Absatz 1 ,schriftliche und mündliche Propaganda, Veranstaltungen, Demonstrationen sowie Aufmärsche, die den Zweck verfolgen, die unteilbare Einheit der türkischen Republik mit ihrem Staatsgebiet und der Nation zu zerstören. Wer dagegen verstößt, wird mit Freiheitsstrafe von einem Jahr bis zu drei Jahren und Geldstrafe bestraft. Bei Rückfall können die verhängten Freiheitsstrafen nicht in Geldstrafen umgewandelt werden. “8 In den Absätzen 2 und 3 waren eigenständige Sanktionen gegen Medienorgane und ihre Verantwortlichen enthalten, wenn die Tat durch die Presse begangen wurde.

Nachdem in der Folgezeit eine umfassende Neugestaltung des TBG vorgenommen wurde, ${ }^{9}$ hat man im Vierten Justizpaket nunmehr einen weiteren Punkt entschärft, der auch in Zusammenhang mit der Frage steht, ob es strafbar sein könnte, wenn man beispielsweise auf den inhaftierten Führer der PKK - wie es auch die Abgeordneten der Kurdenpartei BDP regelmäßig tun - mit „Verehrter Öcalan“ Bezug nimmt oder die Kämpfer der PKK als „Guerillas“ oder „Aufständische“ (isyancılar) bezeichnet. Diesbezüglich verengt der neue Art. 6 Abs. 2 TBG den Anwendungsbereich der Norm in der Gestalt, dass nicht mehr allein auf die bloße Drucklegung oder die Veröffentlichung von Erklärungen einer Terrororganisation abgestellt wird. Vielmehr ist einschränkend erforderlich, dass solche Erklärungen den durch die Terrororganisation erfolgenden Einsatz von Mitteln, die Gewalt oder Drohung enthalten, als legitim erscheinen lassen, verherrlichen oder zum Einsatz solcher Mittel aufmuntern. Auch der neue Art. 7 Absatz 2 TBG greift hinsichtlich der Strafbarkeit der Propaganda für eine terroristische Organisation dieses einschränkende Merkmal auf. Gleiches gilt schließlich für die Strafbarkeit einer propagandistischen Tätigkeit für eine kriminelle Vereinigung (Art. 220 VIII $\left.\mathrm{tStGB}^{10}\right)$.

Auf gleicher Linie liegt eine weitere Änderung im Strafgesetzbuch: Der Straftatbestand der Verherrlichung einer Straftat oder eines Straftäters nach Art. 215 tStGB ist nun nicht mehr als abstraktes, sondern als konkretes Gefährdungsdelikt gestaltet; demnach macht sich wegen entsprechender Verherrlichung nur strafbar, wessen Tat zu einer „offensichtlichen und nahen Gefahr für die öffentliche Ordnung“ führt. Diesbezüglich hatte der Gesetzgeber schon $2005^{11}$ in der geänderten Fassung des $\S 218$ tStGB klargestellt, dass bei Straftaten gegen den öffentlichen Frieden zwar eine Straferhöhung für

5 Gesetz mit der Nummer 3713 vom 12.4.1991, veröffentlicht in Resmî Gazete vom 12.4.1991, Nr. 20843.

6 Art. 19 des Gesetzes mit der Nummer 4928 vom 15.7.2003, veröffentlicht in Resmî Gazete vom 19.7.2003, Nr. 25173.

7 Tellenbach Einführung in das türkische Strafrecht, 2003, S. 99 mit Beispielen aus der früheren Rechtsprechung. Dem weitaus größten Teil der Verfahren lagen Artikel in Zeitungen, Zeitschriften oder Büchern zugrunde. Eine weitere Gruppe betraf Äußerungen von Prominenten bei Pressekonferenzen, Versammlungen oder Konzerten.

8 In der Fassung vom 27.10.1995 durch Art. 1 des Gesetzes mit der Nummer 4126.

9 Gesetz mit der Nummer 5532 vom 29.6.2006, veröffentlicht in Resmî Gazete vom 18.7.2006, Nr. 26232.

10 Gesetz mit der Nummer 5237vom 26.9.2004, veröffentlicht in Resmî Gazete vom 12.10.2004, Nr. 25611.

11 Art. 25 des Gesetzes mit der Nr. 5377 vom 29.6.2005, veröffentlicht in Resmî Gazete vom 8.7.2005, Nr. 25869. 
den Fall einer Tatbegehung durch die Presse erfolgen kann, davon allerdings solche Meinungsäußerungen ausgenommen sind, die „die Grenzen der Nachrichtenübermittlung nicht überschreiten und mit der Absicht getätigt werden, Kritik zu äußern“.

Ebenfalls vor dem Vierten Justizpaket hatte der türkische Gesetzgeber auf die Auswüchse bei der Strafverfolgung wegen eines (vermeintlichen) Verstoßes gegen Art. 301 tStGB reagiert: Nach Absatz 1 dieser Vorschrift wurde bestraft, wer ,das Türkentum, die Republik oder die Große Türkische Nationalversammlung öffentlich verunglimpft"“. ${ }^{12}$ Gleiches galt gemäß Absatz 2 - mit einem im Vergleich um ein Jahr milderen Höchststrafmaß von zwei Jahren Freiheitsstrafe - für die öffentliche Verunglimpfung der Regierung der türkischen Republik, der Justizorgane sowie des Militär- und Sicherheitsapparats des Staates. Wurde die Verunglimpfung des Türkentums durch einen türkischen Staatsbürger im Ausland begangen, war die Strafe um ein Drittel zu erhöhen (Absatz 3). Allerdings stellte das Gesetz in Absatz 4 auch klar, dass „Meinungsäußerungen, die in der Absicht getätigt werden, Kritik zu äußern", nicht bestraft werden dürfen. Dennoch gab es in der darauffolgenden Zeit eine Vielzahl von Ermittlungen, die sich auch gegen namhafte Persönlichkeiten des öffentlichen Lebens richteten. ${ }^{13}$ Aus strafrechtlicher Sicht wurde insbesondere bemängelt, dass die Merkmale „Türkentum“ und „Republik“ zu unbestimmt waren, als dass sie dem Bestimmtheitsgebot (Art. 38 Abs. 1 türkVerf, Art. 7 Abs. 1 EMRK) hätten gerecht werden können. Auf die Kritik in der türkischen Öffentlichkeit und aus europäischen Kreisen reagierte der türkische Gesetzgeber sowohl mit einer inhaltlichen als auch einer prozessualen Änderung: Seit der Neufassung im Jahr 2008 ist zu bestrafen, wer „die türkische Nation, den Staat der türkischen Republik, die Regierung der türkischen Republik und die Justizorgane des Staates öffentlich verunglimpft". Gleiches gilt für die öffentliche Verunglimpfung des Militär- und Sicherheitsapparats des Staates. Die Straferhöhung wegen Verunglimpfung im Ausland durch einen türkischen Staatsbürger wurde restlos gestrichen. In prozessualer Hinsicht ging es in erster Linie darum, das Geschehen nicht in die Hände einzelner, sich für eine „Verteidigung des Türkentums berufener“ Staatsanwälte zu legen. Dementsprechend darf nach dem neu eingefügten ${ }^{14}$ Absatz 4 ein Ermittlungsverfahren wegen Verstoßes gegen Art. 301 tStGB nur mit Zustimmung des Justizministers durchgeführt werden. ${ }^{15}$

\section{Prozessrecht}

Angesichts der eingangs erwähnten Vollzugsdefizite in der türkischen Strafjustiz betrifft der größte Anteil der jüngsten Änderungen den prozessrechtlichen Bereich. Zu erwähnen sind hier vor allem die Schwachstellen in der Praxis, die mit der Anordnung und der Fortdauer der Untersuchungshaft zusammenhängen. In dieser Hinsicht hat sich der

12 In der ursprünglichen Fassung vom 26.9.2004 im Gesetz mit der Nummer 5237.

13 Vgl. www.tagesspiegel.de vom 21.3.2008 „Was steckt hinter dem umstrittenen Paragraf 301?““.

14 Durch Art. 1 des Gesetzes mit der Nummer 5759 vom 30.4.2008, veröffentlicht in Resmî Gazete vom 8.5.2008, Nr. 26870.

15 Ausführlich zur Änderung des Art. 301 tStGB Turhan Türkiye Adalet Akademisi Dergisi 5/2011, S. 286 ff. 
türkische Gesetzgeber weiter um eine solide rechtsstaatliche Gestaltung der betreffenden Vorschrift bemüht, ohne freilich in letzter Konsequenz in die praktische Umsetzung direkt eingreifen zu können. Ebenso von Bedeutung sind aber auch die weiteren Neuerungen im Bereich der Wiederaufnahmegründe sowie einzelner Angeklagtenrechte.

\section{Untersuchungshaft}

\section{a) Anordnung des Haftbefehls}

Zu den chronischen Krankheiten des türkischen Strafprozesses gehört der Umgang mit der Untersuchungshaft. Sie wird oft zu voreilig verhängt und dauert in den meisten Fällen länger als notwendig fort, so dass sie einer vorweggenommenen Strafvollstreckung nahekommt anstatt ihre grundsätzliche Funktion zu erfüllen, die Durchführung des Strafverfahrens und den sich möglicherweise anschließenden Strafvollzug zu sichern. Die in Art. 100 Abs. 1 tStPO ${ }^{16}$ niedergelegten Voraussetzungen für die Anordnung einer Untersuchungshaft sind mit denen des deutschen Strafprozesses $(\S 112$ Abs. $1 \mathrm{dStPO}$ ) im Ausgang identisch: Erforderlich sind Tatsachen, die einen dringenden Tatverdacht begründen, sowie das Vorliegen eines Haftgrunds (Flucht/-gefahr und/oder Verdunklungsgefahr). Ebenso findet sich die Einschränkung, dass Untersuchungshaft nicht angeordnet werden darf, wenn sie zur Bedeutung der Sache und der zu erwartenden Strafe oder Maßregel der Besserung und Sicherung außer Verhältnis steht.

Ein bedeutender Unterschied kommt allerdings hinsichtlich der in Absatz 3 aufgeführten Katalogtaten zum Vorschein, bei denen von einem Haftgrund ausgegangen werden kann (nicht muss!), wenn ein dringender Tatverdacht gegeben ist. Im Unterschied zur parallel gestalteten Regelung in $\S 112$ Abs. 3 dStPO, der lediglich einige wenige Kapitaldelikte enthält, umfasst der Katalog in Art. 100 Abs. 3 tStPO derzeit 16 Delikte von unterschiedlicher Schwere, beispielsweise Völkermord, Folter oder Vergewaltigung, aber auch einfachen Diebstahl oder Verstöße gegen die Strafnormen des Forstgesetzes oder des Gesetzes zum Schutze der Kultur- und Naturgüter. In der Praxis hat sich gezeigt, dass Richter unterschwellig von einer „Verpflichtung zur Verhaftung“ ausgehen, wenn die Katalogtaten einschlägig sind. ${ }^{17}$ Indessen handelt es sich dabei um eine Ermessensvorschrift, die auch und vor allem im Lichte der Unschuldsvermutung (Art. 6 Abs. 2 EMRK) und des Verhältnismäßigkeitsgrundsatzes (mildere Mittel) anzuwenden ist. ${ }^{18}$

Um Auswüchsen gegen das Verhältnismäßigkeitsprinzip bei weniger schwerwiegenden Delikten generell vorzubeugen, bestimmt das Gesetz in Art. 100 Abs. 4 tStPO, dass bei Straftaten, die nur eine Geldstrafe oder ein Strafhöchstmaß von zwei Jahren Freiheitsstrafe vorsehen, ein Untersuchungshaftbefehl nicht erlassen werden darf. Die Grenze von zwei Jahren Freiheitsstrafe fand sich bereits in der ursprünglichen Fassung der

16 Gesetz mit der Nummer 5271 vom 4.12.2004, veröffentlicht in Resmî Gazete vom 17.12.2004, Nr. 25673.

17 So die Feststellung von Öztürk/Erdem Uygulamalı Ceza Muhakemesi Hukuku, 11. Aufl. 2007, S. 561.

18 Nachdrücklich Ünver/Hakeri Ceza Muhakemesi Hukuku 5. Aufl. 2012, S. 389 f.; Großer Senat des Kassationsgerichtshofs, Urteil vom 16.12.2008, 4.MD-225/231. 
Vorschrift. Der Gesetzgeber setzte diese jedoch zwischenzeitlich auf ein Jahr fest; ${ }^{19}$ mit dem Dritten Justizpaket ${ }^{20}$ kehrte man letztlich doch zur Ursprungsfassung zurück.

\section{b) Begründung des Haftbefehls}

Eine effektive Verteidigung gegen einen Haftbefehl ist nur möglich, wenn man sich mit den leitenden Erwägungen des Gerichts auseinandersetzen kann. Dies setzt voraus, dass ein Mindestmaß an Begründung geliefert wird, die sich nicht in einer Wiederholung des Gesetzestextes oder in inhaltsleeren Floskeln erschöpft. Genau dieser fehlerhafte Umgang beim Erlass eines Haftbefehls wird aber der türkischen Justiz regelmäßig angelastet. Es finden sich in der Praxis kaum Beschlüsse, die mehr als ein paar Zeilen enthalten. Ein Beschluss über die Aufhebung eines Haftbefehls wird in der Regel ausführlicher begründet als einer über seinen Erlass. Eine Auseinandersetzung mit dem konkreten Akteninhalt findet nicht statt; die gesetzlichen Merkmale werden demnach mit den Fakten des Einzelfalles nicht in Beziehung gebracht. Vielmehr begnügt man sich mit der Bejahung von einzelnen Punkten, die sich im Gesetz wiederfinden, und lässt offen, welche tatsachenbasierten Erwägungen zum Entschluss geführt haben, dass die Voraussetzungen für den Erlass eines Haftbefehls erfüllt sind. ${ }^{21}$ Damit hängt auch zusammen, dass sich die stichwortartigen Bezugspunkte bei der Aufhebung eines Haftbefehls meistens mit den Erwägungen bei dessen Erlass decken. Sowohl beim Erlass als auch bei der Aufhebung wird also vor allem auf die Schwere der Tat, den Umfang der Beweisaufnahme, den Stand des Verfahrens oder die bisherige Haftdauer abgestellt.

Die Ursachen für diese Missstände liegen nicht im Gesetz. Schon in seiner ursprünglichen Fassung ordnete Art. 101 Abs. 2 tStPO an, dass Entscheidungen über den Erlass oder die Fortdauer eines Haftbefehls sowie die Ablehnung eines Antrags auf Aufhebung des Haftbefehls „mit rechtlichen und tatsächlichen Erwägungen“ zu begründen sind. Nachdem in der Praxis keine Besserung zu beobachten war, hat der Gesetzgeber im Dritten Justizpaket den Versuch unternommen, durch eine umfassendere Beschreibung des Inhalts eines Haftbefehls die Praxis dazu anzuhalten, ihrer Begründungspflicht nicht nur formell, sondern auch materiell nachzukommen. In diesem Sinne schreibt Art. 101 Abs. 2 tStPO nunmehr vor, dass bei Entscheidungen über den Erlass oder die Fortdauer eines Haftbefehls sowie der Ablehnung eines Antrags auf Aufhebung des Haftbefehls Beweise, die den dringenden Tatverdacht, das Vorliegen eines Haftgrundes und die Verhältnismäßigkeit der Untersuchungshaft rechtfertigen, offen anzugeben und mit einer auf konkrete Tatsachen gestützten Begründung zu versehen sind.

19 Art. 11 des Gesetzes mit der Nummer 5353 vom 25.5.2005, veröffentlicht in Resmî Gazete vom 1.6.2005, Nr. 25832.

20 Gesetz mit der Nummer 6352 vom 2.7.2012, veröffentlicht in Resmî Gazete vom 5.7.2012, Nr. 28344.

21 Zu Recht kritisch Centel/Zafer, Ceza Muhakemesi Hukuku, 4. Aufl. 2006, S. 308. 


\section{c) Akteneinsichtsrecht der Verteidigung bei vollzogener Untersuchungshaft}

Eine effektive Verteidigung gegen einen Haftbefehl setzt nicht nur die Kenntnis von Erwägungen voraus, die für das Gericht leitend bei seiner Entscheidung waren, sondern auch ein Mindestmaß an Wissen über den Akteninhalt, auf den sich die gerichtliche Begründung stützt. Dies gilt auch für die Fälle, in denen die Akteneinsicht wegen Gefährdung des Untersuchungserfolgs eingeschränkt wird (Art. 153 Abs. 2 tStPO). Ohne jegliche Kenntnis über die Tatsachenbasis eines Haftbefehls ist die Verteidigung gezwungen - um es bildlich auszudrücken -, im Dunkeln mit geschlossenen Augen auf eine Zielscheibe zu zielen, um diese in der Mitte zu treffen. ${ }^{22}$ Daher hat der EGMR mit Verweis auf Art. 5 IV, 6 I EMRK mehrfach betont, dass der Verteidigung Kenntnis von solchen Aktenteilen zu verschaffen ist, die der Haftentscheidung zugrunde liegen, damit sie sich effektiv gegen den erhobenen Vorwurf verteidigen kann. ${ }^{23}$ Bekanntlich hat der deutsche Gesetzgeber auf diese Rechtsprechung mit der Ergänzung des § 147 II dStPO um die Regelung reagiert, dass ,,dem Verteidiger die für die Beurteilung der RechtmäBigkeit der Freiheitsentziehung wesentlichen Informationen in geeigneter Weise zugänglich zu machen (sind); in der Regel ist insoweit Akteneinsicht zu gewähren.“24 An einer vergleichbaren Regelung fehlt es im türkischen Strafverfahrensrecht; in der Praxis wird die daraus resultierende Verkürzung der Rechte der Verteidigung nachdrücklich beklagt. $^{25}$

22 Dazu Isfen/Arslanbaş Rechtsbrücke/Hukuk Köprüsü 2/2012, S. 167.

23 Vgl. Lamy vs. Belgien, Urteil vom 30.3.1989, Individualbeschwerde-Nr. 10444/83, StV 1993, 283; Garcia Alva vs. Deutschland, Urteil vom 13.2.2001, IndividualbeschwerdeNr. 23541/94, NJW 2002, 2018; Lietzow vs. Deutschland, Urteil vom 13.2.2001, Individualbeschwerde-Nr. 24479/94, NJW 2002, 2013; Schöps vs. Deutschland, Urteil vom 13.2.2001, Individualbeschwerde-Nr. 25116/94, NJW 2002, 2015; Mooren vs. Deutschland, Urteil vom 9.7.2007, Individualbeschwerde-Nr. 11364/03, StV 2008, 475. Ebenso BVerfG NJW 1994, 3219. Ausführlich zu dieser Problematik Erdem Dokuz Eylül Üniversitesi Hukuk Fakültesi Dergisi 1/2004, S. $67 \mathrm{ff}$.

24 BGBl I 2009, S. $2274 \mathrm{ff}$.

25 Allerdings verwarf der EGMR kürzlich die auch auf die Verletzung dieses Rechts gestützte Beschwerde von Tuncay Özkan als unbegründet, vgl. www.radikal.com.tr vom 17.2.2012 „AİHM, tutukluluğu ve şüpheyi makul buldu“. 


\section{d) Dauer der Untersuchungshaft}

Neben der Problematik der vergleichsweise zahlreich ${ }^{26}$ und voreilig verhängten Untersuchungshaft gehört deren zu lange Fortdauer zu den größten Schwachstellen in der türkischen Strafpraxis. Die Gründe für diesen Missstand sind vielfältig. Zunächst fällt auf, dass der türkische Justizapparat chronisch unterbesetzt ist: Bei einer aktuellen Einwohnerzahl von knapp 75 Millionen im Jahr 2011 betrug die Zahl der Richter und Staatsanwälte in den unteren Instanzen der ordentlichen Gerichtsbarkeit 9456 (abzüglich abgeordneter Richter und Staatsanwälte), wobei 5343 Richterstellen (einschließlich der Zivilgerichtsbarkeit) und 4098 Staatsanwaltsstellen zu verzeichnen waren. ${ }^{27}$ Damit hängt unmittelbar zusammen, dass Strafverfahren auch mit Untersuchungshäftlingen gelegentlich für mehrere Monate unterbrochen werden (müssen). ${ }^{28}$ Des Weiteren werden in der Praxis oft umfangreiche Ermittlungsverfahren mit zahlreichen Beschuldigten durchgeführt: Da das türkische Strafverfahren das Legalitätsprinzip strikt anwendet und sehr wenige Mittel zur alternativen Verfahrenserledigung im Wege des Opportunitätsprinzips zur Verfügung stehen, sind Anklagen gegen mehrere hundert Beschuldigte mittlerweile keine Seltenheit. Hinzu kommt eine großzügige Anwendung des Instituts der Verfahrensverbindung, so dass beispielsweise im Ergenekon-Prozess ${ }^{29}$ etwa 20 Anklagen trotz unterschiedlichster Ausgangslage zusammengeführt wurden, weil den beschuldigten Personen ein ganz weit gefasster gemeinsamer Handlungsplan unterstellt wird. Das alles hat dazu geführt, dass im erwähnten Verfahren, das seit Juli 2008 immer noch in der ersten Instanz andauert und bei dem zahlreiche Beschuldigte sich seit Sommer 2007 in Untersuchungshaft befinden, mittlerweile 275 Angeklagte (davon 66 in

26 In den letzten Jahren ist jedoch ein entgegengesetzter Trend zu beobachten: Waren im Jahr 2002 noch etwa $41 \%$ aller Insassen einer Justizvollzugsanstalt Untersuchungshäftlinge, so schnellte diese Zahl 2006 zwar auf $49 \%$ hoch, nahm aber seitdem stetig ab. Die aktuellsten Zahlen für das Jahr 2012 weisen zum Stichtag insgesamt 31.707 Untersuchungshäftlinge aus, was bei 104.313 Verurteilten einem Verhältnis von ca. $23 \%$ entspricht (Adalet Bakanlığı Ceza ve Tevkif Evleri Genel Müdürlüğü, Ceza İnfaz Kurumlarının Yıllara Göre Mevcutları, abgerufen am 30.4.2013 unter www.cte.adalet.gov.tr „İstatistikler“). Bei vergleichbarer Bevölkerungszahl lag die aktuellste Zahl (für das Jahr 2011) der Untersuchungshäftlinge in Deutschland bei 10.793 gegenüber insgesamt 68.099 Verwahrten, was einer Quote von etwa $16 \%$ entspricht (Statistisches Bundesamt, Fachserie 10 Reihe 1 Rechtspflege 2012, 2013, S. 91).

27 Hâkimler ve Savcılar Yüksek Kurulu 2011 Y1lı Faaliyet Raporu, 2012, S. 28. Zum vergleichbaren Zeitpunkt verfügte Deutschland bei etwa 82 Millionen Einwohnern über 14.801 Richterstellen und 5249 Staatsanwaltsstellen in der ordentlichen Gerichtsbarkeit (Statistisches Bundesamt, Fn. 26, S. 15).

28 Insofern fehlt es im türkischen Strafverfahrensrecht an einer Regelung wie in $\S 229$ Abs. 1 und 2 dStPO, die eine grundsätzliche Unterbrechungsgrenze von drei Wochen bzw. einem Monat enthält.

29 Mit der Bezeichnung „Ergenekon“ wird auf eine nationalistische Untergrundbewegung (,tiefer Staat") Bezug genommen, die aus ehemaligen Militärs, Bürokraten, Juristen, Geschäftsleuten, Politikern (darunter auch gewählte Abgeordnete der laufenden Legislaturperiode, die sich weiter in Untersuchungshaft befinden, dazu näher Çelik/Tekkoyun Türkiye Adalet Akademisi Dergisi 6/2011, S. 414 ff.) und Journalisten bestanden und ab 2003 durch Terrorakte sowie Desinformation den Sturz der Regierung des Premierminister Erdoğan betrieben haben soll. 
Untersuchungshaft) abgeurteilt werden. ${ }^{30} \mathrm{Im}$ anderen öffentlichkeitswirksamen $\mathrm{Ba}$ lyoz-Prozess, der sich um den Vorwurf des Putschversuchs von Generälen gegen die Regierung des Premierministers Erdoğan dreht, waren es sogar 365 Angeklagte (davon 265 in Untersuchungshaft), die mittlerweile zu hohen Freiheitsstrafen verurteilt wurden. Vor diesem Hintergrund sind Anklagen auf mehreren tausend Seiten einschließlich hunderter Beweismittelordner sowie Urteilsbegründungen im Umfang von ebenfalls mehreren tausend Seiten keine Rarität.

Mit Blick auf den Beschleunigungsgrundsatz in Art. 5 Abs. 3 EMRK ${ }^{31}$ legen die dargestellten Schwierigkeiten in der Praxis den Gedanken einer gesetzlichen Konstituierung von Höchstgrenzen nahe. In der Tat enthält die türkische Strafprozessordnung solche Schranken, die allerdings in problematischen Fällen oft keine Wirkung entfalten: Nach Art. 102 Abs. 1 tStPO darf Untersuchungshaft bei Delikten, die nicht in die Zuständigkeit der Gerichte für schwere Delikte (Ağır Ceza Mahkemesi) fallen, höchstens für die Dauer eines Jahres angeordnet werden; erforderlichenfalls kann mit entsprechender Begründung eine Verlängerung bis zu sechs Monaten erfolgen. Gemäß Absatz 2 beträgt die Höchstdauer der Untersuchungshaft bei Delikten, die in die Zuständigkeit der Gerichte für schwere Delikte (Ağır Ceza Mahkemesi) fallen, zwei Jahre; auch hier kann mit entsprechender Begründung eine Verlängerung erfolgen, wobei die Dauer der Verlängerung drei Jahre nicht überschreiten darf. Somit eröffnet sich in den Konstellationen des Absatzes 2 eine Höchstdauer von fünf Jahren, was an sich grenzwertig erscheint. Allerdings wird diese Grenze nochmals verdoppelt auf zehn Jahre, wenn nach Art. 10 V TBG bestimmte Delikte gegen die Staatssicherheit im Raum stehen (wie bei Ergenekon). Ein so langer Zeitraum zwischen Erlass des Haftbefehls und gerichtlicher Entscheidung in der Hauptsache dürfte mit dem in Art. 5 Abs. 3 EMRK niedergelegten Recht auf ein beschleunigtes Verfahren bei Freiheitsentzug in aller Regel nicht zu vereinbaren sein. ${ }^{32}$ Bedauerlicherweise haben sowohl das Dritte als auch das Vierte Justizpaket an diesen Rahmenbedingungen des Art. 102 Abs. 2 tStPO sowie des Art. $10 \mathrm{~V}$ TBG nichts geändert.

Angesichts der teils sehr langen Verfahrensdauer in der Türkei gewinnt in diesem Kontext die Frage an Bedeutung, ob diese Grenzwerte das Rechtsmittelverfahren umfassen. Mit anderen Worten: Gilt der in erster Instanz verurteilte Angeklagte, der Revision eingelegt hat, noch als Untersuchungshäftling, so dass er bei Erreichen der Obergrenzen (vorläufig) entlassen werden müsste? Die türkische Praxis verneint dies mehrheitlich mit Verweis auf die Rechtsprechung des EGMR im Fall Wemhoff vs. Deutschland, wonach bei der Auslegung des Art. 5 Abs. 3 EMRK ,die Tatsache nicht übersehen werden (kann), dass die Schuld einer während des Berufungs- oder Revisionsverfahrens in Haft gehaltenen Person in einem Prozess, der den Anforderungen des Art. 6 entsprach, festgestellt worden ist. “33 Eine solche Auslegung mag für die Einhaltung der Grenzen des Art. 102 tStPO, $10 \mathrm{~V}$ TBG ein willkommener Rettungsanker sein; allerdings müssen sich die Schranken ihrerseits an den Vorgaben des Art. 5 Abs. 3, Art. 6 Abs. 1 EMRK

30 www.sabah.com.tr vom 11.12.2012 „'Ergenekon'da sona gelindi“.

31 Dazu pars pro toto C. gegen Deutschland, Urteil vom 29.7.2004, IndividualbeschwerdeNr. 49746/99, NJW 2005, 3125.

32 Ebenso Özen/Güngör/Ergün Ankara Barosu Dergisi 4/2010, S. 183.

33 Urteil vom 25.4.1968, Individualbeschwerde-Nr. 2122/64, Ziffer 9. Mit weiteren Beispielen aus der EGMR-Rechtsprechung Özen/Güngör/Ergün Ankara Barosu Dergisi 4/2010, S. 184 f. 
messen lassen. Diesbezüglich hat aber der EGMR beispielsweise im Fall Cahit Demirel vs. Türkei bereits entschieden, dass eine Untersuchungshaftdauer von sechs Jahren und vier Monaten vor gerichtlicher Verurteilung in einer Staatssicherheitsangelegenheit einen Verstoß gegen Art. 5 Abs. 3 EMRK darstellt. ${ }^{34}$ Nach den Umständen des Einzelfalles kann jedoch eine weitaus geringe Haftdauer einen Verstoß gegen den Beschleunigungsgrundsatz begründen, zum Beispiel ein Jahr und acht Monate im Fall Bykov vs. Russland, der den Vorwurf einer Anstiftung zum Mord zum Gegenstand hatte. ${ }^{35}$ Insofern kann die Betrachtungsweise nach festgelegten zeitlichen Höchstgrenzen leicht dazu führen, die Rechtmäßigkeit der Untersuchungshaftdauer maßgeblich nach diesen Vorgaben zu beurteilen. Das wäre jedoch eine fatale Verkennung der rechtlich ausschlaggebenden Gesichtspunkte, die der Beurteilung der Rechtfertigungsfähigkeit staatlichen Freiheitsentzugs bei Fortgeltung der Unschuldsvermutung zugrunde gelegt werden müssen.

\section{e) Mildere Maßnahmen anstelle der Untersuchungshaft}

In Anbetracht der zunehmenden Kritik in der türkischen Öffentlichkeit an der Untersuchungshaftdauer bei überlangen Strafverfahren hat der türkische Gesetzgeber im Dritten Justizpaket den Versuch unternommen, größere Handlungsspielräume für Gerichte zu schaffen, was die Anordnung von milderen Mitteln angeht. Diesbezüglich sah Art. 109 Abs. 1 tStPO schon in seiner ursprünglichen Fassung vor, dass bei Ermittlungsverfahren, in denen es um Delikte mit einer Strafobergrenze von drei Jahren Freiheitsstrafe geht, anstelle der Untersuchungshaft gegen den Beschuldigten Maßnahmen der justiziellen Kontrolle (Adlî Kontrol) auf ihn angewendet werden können. Mit dem Dritten Justizpaket hat der Gesetzgeber nunmehr die Beschränkung auf die erwähnte Strafobergrenze restlos abgeschafft, so dass selbst bei schwersten Delikten justizielle Kontrolle erfolgen kann. Möglich sind etwa Ausreiseverbote, Meldeauflagen oder Verhaltensauflagen wie z.B. das Verbot, bestimmte Orte aufzusuchen oder die Unterkunft zu verlassen (Art. 109 Abs. 3 tStPO).

\section{f) „Freiheitsrichter“}

Der unbefriedigende Umgang der Richterschaft mit der Verhängung von Untersuchungshaft hat den türkischen Gesetzgeber im Dritten Justizpaket dazu bewogen, einen ersten Schritt zu einer Neukonzeption der Entscheidungsprozesse im Ermittlungsverfahren zu machen. Bisher war es nicht ungewöhnlich, dass Richter als Folge der Geschäftsverteilung Zwangsmaßnahmen im Ermittlungsverfahren anordneten und anschließend in der Hauptsache gegen denselben Beschuldigten auftraten. Dies führte unweigerlich zu Zweifeln an der Unvoreingenommenheit dieser beteiligten Richter. Die neue Regelung des Art. 10 Abs. 3c TBG sieht hingegen - zunächst für einen eingegrenzten Bereich - vor, dass richterliche Entscheidungen in Ermittlungsverfahren, die die Straftatbestände des TBG und (i.V.m. Abs. 4) weitere schwere Delikte aus dem

34 Urteil vom 7.7.2009, Individualbeschwerde-Nr. 18623/03.

35 Urteil vom 10.3.2009, Individualbeschwerde-Nr. 4378/02. 
Kern- und Nebenstrafrecht betreffen, von Richtern gefällt werden, die allein für diese Aufgabe zuständig sind. Auf diese Weise sollen die grundrechtsrelevanten Entscheidungen über Zwangsmaßnahmen vom „Alltagsgeschäft“ der Strafgerichte entkoppelt und eigens dafür vorgesehenen Richtern übertragen werden, von denen man sich ein besonderes Gespür für Freiheitsrechte des Beschuldigten verspricht. Passend zu dieser Erwartungshaltung werden diese Ermittlungsrichter - in Anlehnung an das französische Vorbild ,juge des libertés et de la détention“36 - als „Freiheitsrichter“ (Özgürlük Hâkimi) bezeichnet. Auch wenn diese personelle Trennung (noch) nicht das gesamte Strafverfahren erfasst, scheint der eingeschlagene Weg ein zustimmungswürdiger zu sein. Allerdings wird auch hier erst die praktische Handhabung zeigen, ob es den Freiheitsrichtern gelingt, die Rechte des Beschuldigten mit dem Interesse der Allgemeinheit an effektiver Strafverfolgung in Einklang zu bringen.

\section{Erweiterung der Wiederaufnahmegründe}

Die Bedeutung der EMRK für die türkische Strafrechtspraxis zeigt sich insbesondere darin, dass im Vierten Justizpaket die Transformationsmöglichkeiten einer für den betroffenen Bürger vorteilhaften Entscheidung des EGMR um weitere Punkte ergänzt wurden. Neben der bisherigen generellen Bestimmung in Art. 311 Abs. $1 \mathrm{f} \mathrm{tStPO}$ sieht nunmehr auch Art. 64 Abs. 11 des Gesetzes über den Hohen Militärverwaltungsgerichtshof vor, dass eine Wiederaufnahme des Verfahrens beantragt werden kann, wenn durch den EGMR rechtskräftig festgestellt wird, dass die betreffende Entscheidung gegen die EMRK oder ihre Zusatzprotokolle verstößt und sie auf diesem Verstoß beruht. Ebenfalls neu ist die Regelung in Art. 172 Abs. 3 tStPO, wonach das Ermittlungsverfahren auf Antrag wieder aufgenommen werden muss, wenn durch den EGMR rechtskräftig festgestellt wird, dass eine staatsanwaltschaftliche Einstellungsentscheidung ergangen ist, ohne dass effektiv ermittelt wurde.

\section{Verteidigung in einer anderen Sprache als der türkischen}

Der erwähnte Paradigmenwechsel in der Frage der Kurdenproblematik ${ }^{37}$ erlaubt den handelnden Akteuren Gestaltungsräume, die noch vor einigen Jahren undenkbar gewesen oder gar als Straftat ausgelegt worden wären. Kennzeichnend für diese Momentaufnahme der türkischen Politik und Gesellschaft ist beispielsweise das Eingeständnis des (ehemaligen) Generalstaatsanwalts vom Diyarbakır, einer Provinz im Südosten der Türkei mit hohem kurdischem Bevölkerungsanteil, der 2011 auf einer Feierlichkeit an der heimischen Universität gemeinsam mit anderen Anwesenden kurdische Lieder angestimmt und dabei eingestanden hat, dass er in der gleichen Situation im Jahr 2006 die Veranstaltung aus Protest verlassen und dafür gesorgt hätte, dass alle Beteiligten verhaftet werden. ${ }^{38}$ Dieser Stimmungswandel hat im Rahmen des „Lösungsprozesses“ und der damit zusammenhängenden Reformbewegungen in der Justiz auch dazu geführt,

36 Art. 137-1 der französischen Strafprozessordnung.

37 Siehe unter I.

38 www.aksam.com.tr vom 3.6.2011, „2006'da olsa hepinizi tutuklatırdım'“. 
dass zwischen dem Dritten und dem Vierten Justizpaket ein weiteres Gesetz ${ }^{39}$ verabschiedet wurde, das den mündlichen Verteidigungsvortrag des Angeklagten in einer anderen Sprache als der türkischen ermöglicht. Ersichtlich geht es hier in erster Linie um kurdisch stämmige Angeklagte, auch wenn es nicht immer offen zur Sprache gebracht wird. Bis dahin erlaubten die Strafgerichte kurdisch stämmigen Angeklagten nicht, sich auf kurdisch zu verteidigen, weil in der Regel davon ausgegangen wurde, dass diese als Bürger der Türkischen Republik die türkische Sprache beherrschten. Selbstverständlich war es ausländischen Angeklagten, die der türkischen Sprache nicht mächtig waren, schon immer möglich, sich der Hilfe eines Dolmetschers zu bedienen. Allerdings stand diese Möglichkeit gemäß Art. 202 Abs. 1 tStPO unter dem Vorbehalt, dass der Angeklagte „nicht in der Lage ist, sein Anliegen im ausreichenden Maße auf türkisch vorzutragen“. Bei kurdisch stämmigen Angeklagten verneinten die Gerichte das Vorliegen dieses Erfordernisses, was im Einzelfall zu deutlichen Spannungen mit der Garantie des Art. 6 Abs. 3 e EMRK führte. Nunmehr sieht Art. 202 Abs. 4 tStPO vor, dass „darüber hinaus“ (d.h. auch wenn ausreichende Kenntnisse der türkischen Sprache gegeben sind, so dass der Grundfall des Absatzes 1 nicht eingreift) der Angeklagte zumindest seinen auf Verlesung der Anklage und dem staatsanwaltschaftlichen Plädoyer folgenden mündlichen Verteidigungsvortrag in einer Sprache halten darf, in der er sich besser ausdrücken kann. Dabei kann er seinen Dolmetscher aus einer von den Justizbehörden erstellten Liste frei auswählen. Allerdings werden die entstehenden Dolmetscherkosten nicht von staatlichen Stellen getragen. Einen möglichen Widerspruch zu Art. 6 Abs. 3 e EMRK (unentgeltliche Unterstützung) wegen der Nicht-Übernahme der Dolmetscherkosten nimmt der türkische Gesetzgeber offenbar in Kauf, weil er weiterhin davon ausgeht, dass kurdisch stämmige Angeklagte die türkische Sprache ausreichend beherrschen, so dass die unentgeltliche Beiordnung eines Dolmetschers für eine effektive Verteidigung nicht zwingend erscheint. Eine solche pauschale Betrachtungsweise ist jedoch nicht frei von Bedenken.

\section{Ausblick}

Der kurze Blick auf die jüngsten Reformen in der türkischen Strafjustiz hat deutlich gemacht, dass die Türkei seit gut einem Jahrzehnt einen tiefgreifenden Wandel erlebt. Anfänglich mag die EU-Annäherung eine bedeutende Triebkraft in dieser Entwicklung dargestellt haben; mittlerweile hat sich aber der Reformwille von dieser vagen EUPerspektive abgekoppelt und weist eine eigene Dynamik auf. Allerdings sind die Anwender noch davon entfernt, sich trotz der beachtlichen Fortschritte auf einem Niveau zu bewegen, das dem umfassenden Erneuerungsanspruch gerecht werden könnte. Man denke an die Auswüchse rund um den „Gezi-Park“. Mit dem Dritten und Vierten Justizpaket fordert der türkische Gesetzgeber wieder einmal die Praxis regelrecht heraus, indem er die normative Vorlage für eine freiheitlichere Strafrechtsordnung liefert. Es bleibt abzuwarten, ob diese Herausforderung angenommen wird.

39 Gesetz mit der Nummer 6411 vom 24.1.2013, veröffentlicht in Resmî Gazete vom 31.1.2013, Nr. 28545. 